\title{
Erratum
}

\section{Common Fixed Point Theorems for Hybrid Pairs of Occasionally Weakly Compatible Mappings Satisfying Generalized Contractive Condition of Integral Type Revisited}

\author{
M. Abbas ${ }^{1}$ and B. E. Rhoades ${ }^{2}$ \\ ${ }^{1}$ Centre for Advanced Studies in Mathematics and Department of Mathematics, \\ Lahore University of Management Sciences, Lahore 54792, Pakistan \\ ${ }^{2}$ Department of Mathematics, Indiana University, Bloomington, IN 47405, USA
}

Correspondence should be addressed to B. E. Rhoades, rhoades@indiana.edu

Received 3 September 2008; Accepted 30 September 2008

Copyright (c) 2008 M. Abbas and B. E. Rhoades. This is an open access article distributed under the Creative Commons Attribution License, which permits unrestricted use, distribution, and reproduction in any medium, provided the original work is properly cited.

We are indebted to Valeriu Popa for pointing out our error in [1]. In looking again at the paper, we came up with the following example.

Let $X=[0,1]$ with the usual metric, and define $f=g: X \rightarrow X, T: X \rightarrow C B(X)$ by $f x=1-x, T x=\{0,1\}$. Since $f=g$, every point is a coincidence point, and $f T x=T f x$. Also, $H(T x, T y)=0$ for all $x$ and $y$, and $d(f x, g y) \neq 0$ for $x \neq y$, so $f$ and $T$ satisfy the hypotheses of all theorems and corollaries in [1], but $f$ and $T$ have no common fixed point.

Thus, it is not surprising that there are a number of papers involving hybrid pairs in which the conclusion of the theorems is not a common fixed point, but a common coincidence point (see, e.g., [2-10]). To obtain a common fixed point, a number of theorems assume the strong condition that the common coincidence point is also a fixed point of one of the maps.

\section{References}

[1] M. Abbas and B. E. Rhoades, "Common fixed point theorems for hybrid pairs of occasionally weakly compatible mappings satisfying generalized contractive condition of integral type," Fixed Point Theory and Applications, vol. 2007, Article ID 54101, 9 pages, 2007.

[2] A. Constantin, "Coincidence point theorems for multivalued contraction mappings," Mathematica Japonica, vol. 36, no. 5, pp. 925-933, 1991.

[3] M. Imdad, A. Ahmad, and S. Kumar, "On nonlinear nonself hybrid contractions," Radovi Matematički, vol. 10, no. 2, pp. 233-244, 2001.

[4] T. Kamran, "Coincidence and fixed points for hybrid strict contractions," Journal of Mathematical Analysis and Applications, vol. 299, no. 1, pp. 235-241, 2004.

[5] I. Kubiaczyk and B. Deshpande, "Coincidence point for noncompatible multivalued maps satisfying an implicit relation," Demonstratio Mathematica, vol. 39, no. 4, pp. 855-862, 2006. 
[6] S. V. R. Naidu, "Fixed points and coincidence points for multimaps with not necessarily bounded images," Fixed Point Theory and Applications, vol. 2004, no. 3, pp. 221-242, 2004.

[7] H. K. Pathak and S. N. Mishra, "Coincidence points for hybrid mappings," Rostocker Mathematisches Kolloquium, no. 58, pp. 67-85, 2004.

[8] S. L. Singh and Giniswamy, "Concidences and fixed point theorems for single valued and multivalued maps," Fixed Point Theory and Applications, vol. 2004, no. 5, pp. 127-139, 2004.

[9] S. L. Singh, K. S. Ha, and Y. J. Cho, "Coincidence and fixed points of nonlinear hybrid contractions," International Journal of Mathematics and Mathematical Sciences, vol. 12, no. 2, pp. 247-256, 1989.

[10] S. L. Singh and S. N. Mishra, "Coincidences and fixed points of nonself hybrid contractions," Journal of Mathematical Analysis and Applications, vol. 256, no. 2, pp. 486-497, 2001. 